\title{
Isolation Circuits Based on Metamaterial Transmission Lines for Multiplexers
}

\author{
(Invited Paper)
}

\author{
Hanseung Lee $\cdot$ Tatsuo Itoh*
}

\begin{abstract}
Multiplexers based on isolation circuits made of metamaterial lines are proposed and studied. The new approach provides unique advantageous features beneficial to system designer. For instance, there is no need to modify the filters used in multiplexers. Also, the design process is straightforward. In this paper, two types of multiplexers based on metamaterial isolation circuits are presented, and their operation concepts are explained. Also, theories and design process of isolation circuits are presented to help readers design and fabricate proposed multiplexers. For verifying the concepts, two types of triplexers and two types of quadruplexers are designed and fabricated. All filters used in the multiplexers are commercial surface acoustic wave filters. The measured results are well matched with the simulation results.
\end{abstract}

Key Words: Composite Right/Left Handed, Double-Lorentz, Isolation Circuit, Multiplexer, Transmission Line.

\section{Introduction}

Many modern communication systems are required to handle multiple frequency bands. If a base station for a mobile phone can deal with two or three communication standards, the price of its construction will be lowered with concomitant benefits for customers. Multiband is already intrinsic to cell phone designs for handling cellular, global positioning system (GPS), and WiFi bands. In order to support such multiband systems, a multiplexer is necessary if one antenna covers all the designated spectra and thus becomes an essential component. For multiplexers, many design concepts have been investigated and categorized by star junction type and manifold junction type [1-3]. The first star junction type multiplexer is based on synthesizing singly terminated channel filters [4]. After one decade, another method to synthesize doubly terminated filters for star junction type multiplexers was proposed [5]. In the recent approach [6], a general polynomial model is used for filters, and filters are connected with a complex load. The rapid development of manifold junction type multiplexers was caused by the advent of satellite communication systems [7]. In the initial stage, the analytic approach to design the filters, having proper interaction with other filters on the same manifold, was investigated [8]. However, recent manifold junction type multiplexers are mostly designed with optimization methods with the increased computer power [9]. In both star junction and manifold junction type multiplexers, modification of filters and a complex optimization process are generally required. However, the use of a commercial filter without modification, and a simple multiplexer design method are occasionally necessary for a system designer. The simplest multiplexer satisfying such demand is shown in Fig. 1(a). The diplexer is made of two isolation circuits, and each isolation circuit consists of a conventional righthanded transmission line (RH TL) connected to a filter. An isolation circuit isolates itself from the input port at the center frequency of the other channel. Hence the signal passes through one isolation circuit while it is rejected by the other. This procedure cannot be extended simply to a multiplexer containing three or more channels. Since a conventional TL can provide one phase response at a target frequency, an isolation circuit can be designed to have the isolation characteristic at only one frequency.

If a TL can have a multiple phase property, it can realize a multiband isolation circuit and result in a channel extension of the multiplexer based on isolation circuits.

Manuscript received August 27, 2013 ; Revised September 5, 2013 ; Accepted September 10, 2013. (ID No. 20130827-01J)

Department of Electrical Engineering, University of California at Los Angeles (UCLA), CA, USA.

${ }^{*}$ Corresponding Author : Tatsuo Itoh (e-mail: itoh@seas.ucla.edu)

This is an Open-Access article distributed under the terms of the Creative Commons Attribution Non-Commercial License (http://creativecommons.org/licenses/ by-nc/3.0) which permits unrestricted non-commercial use, distribution, and reproduction in any medium, provided the original work is properly cited. 
This characteristic has been found in a metamaterial TL. A composite right/left-handed (CRLH) metamaterial TL has a dual-band characteristic [10]. A double-Lorentz (DL) TL has six parameters, and it satisfies six conditions for a tri-band characteristic [11].

In this paper, two types of multiplexers based on isolation circuits are presented. One consists of isolation circuits using same type of TLs, and it can be categorized as the star junction type. On the other hand, the other type uses both RH TLs and multiband TLs for making isolation circuits, and its junction shape is similar to a manifold junction. First, the concepts of multiplexers are explained, and the theory for designing an isolation circuit is investigated. The simulated and measured results of the proposed multiplexers are also provided.

\section{II . Concept of Proposed Multiplexer}

The diplexer shown in Fig. 1(a) is the starting point for a multiplexer based on isolation circuits. If it is necessary to increase one more channel, an additional isolation circuit can be connected to the junction point like shown in Fig. 1(b), and each circuit should have opencircuit input impedance at the passing frequencies of other circuits [12]. For satisfying this condition, a CRLH TL having dual band characteristic is necessary. In a similar manner, a four-channel multiplexer or a quadruplexer can be realized by connecting four tri-band isolation circuits composed of DL TLs having tri-band characteristic [13].

In the concept of a star junction multiplexer, all isolation circuits are composed with same type of TL. For example, a star junction triplexer is composed with three CRLH TLs followed by three filters. The multiplexer shown in Fig. 1(c) also consists of multiband TLs and filters for multiband isolation circuits. However, one special isolation circuit composed with RH TLs connecting with a filter exists in this type of multiplexers [14, 15]. This special isolation circuit also has multiband characteristic. The mechanism is as follows: 1) at frequency $f_{1}$, the first isolation circuit consisting of the $f_{1}$ filter allows signal path. Meanwhile, other isolation circuits composed with multiband TLs are disconnected from junctions, and the combined phase response of $\mathrm{RH}$ TLs (from $T L_{1}$ to $T L_{N-1}$ ) is already determined for the isolation circuits consisting of RH TLs (from $T L_{1}$ to $T L_{N}$ ) and the $f_{N}$ filter having infinite input impedance. 2) At frequency $f_{N-1}$, except the isolation circuit having the $f_{N-1}$ filter, all isolation circuits consisting of multiband TLs have infinite input impedance. Also the isolation circuit consisting of the RH $T L_{N-1}$ and the $f_{N}$ filter

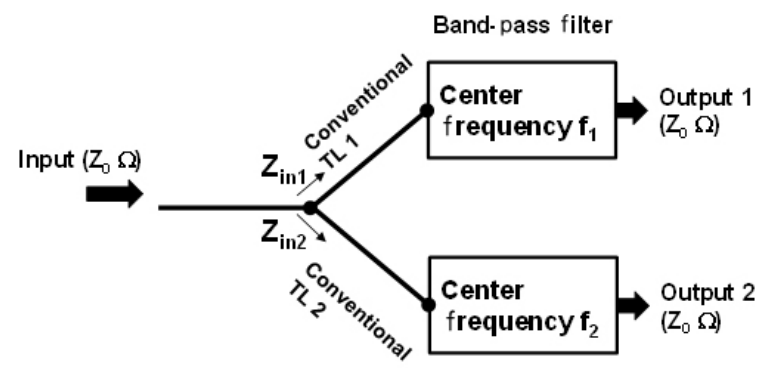

Operation concept,
At $\mathrm{f}_{1}$,
At $\mathrm{f}_{2}$,

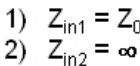
1) $Z_{\text {in1 } 1}=\infty$

(a)

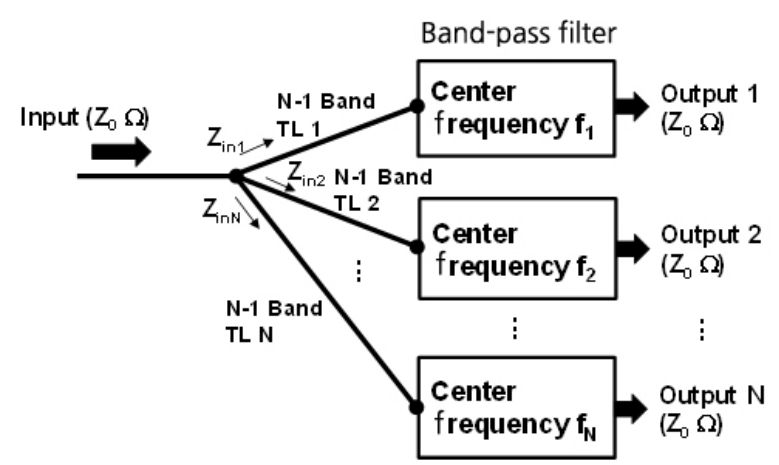

Operation concept,
At $\mathrm{f}_{1}$,
At $\mathrm{f}_{2}$,
At $\mathrm{f}_{\mathrm{N}}$,
1) $Z_{\text {in1 }}=Z_{0}$
1) $Z_{\text {in } 1}=\infty$
2) $Z_{\text {in } 2}=\infty$
2) $Z_{\text {in2 } 2}=Z_{0}$
N) $\mathrm{Z}_{\mathrm{inN}}=\infty$
N) $Z_{\text {inN }}=\infty$
1) $Z_{\text {in1 } 1}=\infty$
2) $Z_{\text {in } 2}=\infty$
N) $\mathrm{Z}_{\mathrm{inN}}=\mathrm{Z}_{0}$

(b)

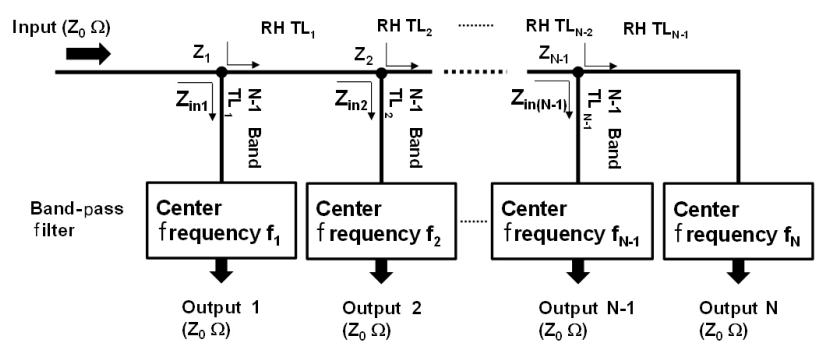

Operation concept,
At $\mathrm{f}_{1}, \quad$ At $\mathrm{f}_{2}$,
At $\mathrm{f}_{\mathrm{N}-1}, \quad$ At $\mathrm{f}_{\mathrm{N}}$,
$\begin{array}{ll}\text { 1) } Z_{\text {in } 1}=Z_{0} & \text { 1) } Z_{\text {in1 } 1}=\infty\end{array}$
$\begin{array}{ll}\text { 1) } Z_{\text {in } 1}=\infty & \text { 1) } Z_{\text {in1 } 1}=\infty \\ \text { 2) } Z_{\text {in } 2}=\infty & \text { 2) } Z_{\text {in } 2}=\infty\end{array}$
2) $Z_{\text {in } 2=\infty}$ 2) $Z_{\text {in } 2}=Z_{0}$
$\vdots:$
N-1) $\left.Z_{\text {in }(N-1)}=\infty \quad N-1\right) Z_{\text {in }(N-1)}=\infty$
$\begin{array}{ll}\text { N) } Z_{1}=\infty & \text { N) } Z_{2}=\infty\end{array}$

(c)

Fig. 1. Diagrams and operation concepts of multiplexers based on isolation circuit: (a) diplexer, (b) star-junction multiplexer, and (c) manifold-junction multiplexer. RH $\mathrm{TL}=$ right-handed transmission line. 
is isolated from the junction. 3) At frequency $f_{N}$, all isolation circuits composed with multiband TLs are isolated from junctions, and the $f_{N}$ filter is directly connected with the input through RH TLs allowing signal path.

In the diagrams of Fig. 1, the frequencies from $f_{1}$ to $f_{N}$ do not need to be in ascending order.

\section{Theory for Isolation Circuit Design}

The method to determine a proper phase response of a TL for an isolation circuit and the theory of a multiband metamaterial TL are discussed in this section.

\section{Required Phase Response for Isolation Circuit}

Fig. 2(a) represents an isolation circuit comprising a TL connected to a filter. For simplicity, a case dealing with only one phase is considered. In Fig. 2(b) and (c), each triangle on the Smith chart shows an input impedance of a filter at target frequency to be isolated. In both cases, the real part of the impedance $(\alpha)$ is assumed zero. Fig. 2(b) and (c) represent a positive imaginary impedance and a negative imaginary impedance, respectively. Since the input impedance is not infinite, a connected TL is required to rotate the triangle to the open position, represented by a hollow circle on the Smith chart, for an isolation characteristic. Two rotations are possible in both cases. Clockwise rotation represents a $\mathrm{RH}$ phase response or a phase delay, $\phi_{1}$, having a nega-

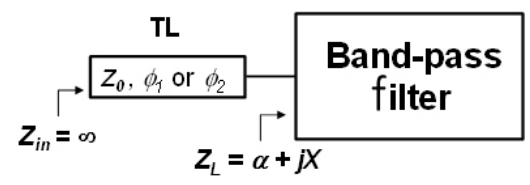

(a)

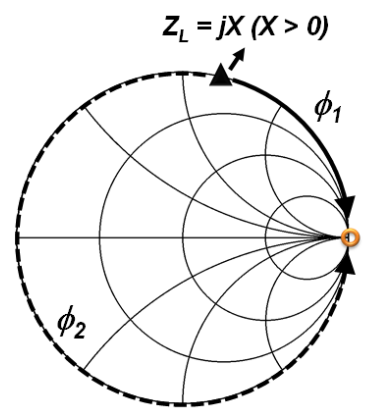

(b)

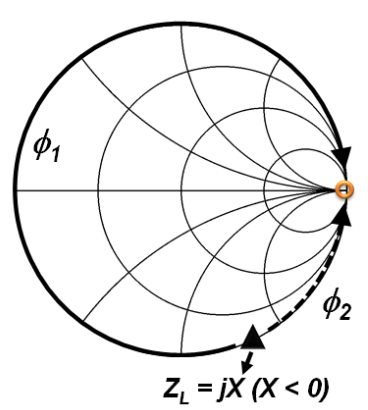

(c)
Fig. 2. (a) An isolation circuit diagram, (b) an example of the input impedance $Z_{L}$ shown on the Smith chart (case $1, X>0$ and $\alpha=0$ ), and (c) an example of the input impedance $Z_{L}$ shown on the Smith chart (case 2, $X<0$ and $\alpha=0$ ). $\mathrm{TL}=$ transmission line. tive value. A left-handed ( $\mathrm{LH})$ phase response or a phase advance, $\phi_{2}$, having a positive value, is represented as a counter-clockwise rotation on the Smith chart. If the real part of the input impedance of a filter or a multiplexer $(\alpha)$ is very small or zero, the transmission line impedance equation can be used to solve the required phase response [16].

$$
Z_{\text {in }}=Z_{0} \frac{Z_{L}+j Z_{0} \tan \left(-\phi_{1,2}\right)}{Z_{0}+j Z_{L} \tan \left(-\phi_{1,2}\right)}
$$

Since the input impedance of the circuit $Z_{i n}$ should be infinite, the denominator of (1) is zero, and the required phase response, $\phi_{1,2}$, is

$$
\begin{aligned}
& \phi_{1}= \begin{cases}-\arctan \left(\mathrm{Z}_{0} / \mathrm{X}\right), & \mathrm{X}>0 \\
-\pi-\arctan \left(\mathrm{Z}_{0} / \mathrm{X}\right), & \mathrm{X}<0\end{cases} \\
& \phi_{2}= \begin{cases}\pi-\arctan \left(\mathrm{Z}_{0} / \mathrm{X}\right), & \mathrm{X}>0 \\
-\arctan \left(\mathrm{Z}_{0} / \mathrm{X}\right), & \mathrm{X}<0\end{cases}
\end{aligned}
$$

These equations can be simplified to a more general form:

$$
\phi=n \pi-\arctan \left(\mathrm{Z}_{0} / \mathrm{X}\right)+\psi
$$

where $n$ is the integer and $Z_{0}$ is the characteristic impedance of the transmission line. Here, $\Psi$ is the adjusting constant to obtain a realistic value near open-circuit position [13]. A more rigorous equation considering the term $\alpha$ is also represented as

$$
\phi=n \pi-\frac{\arg \left\{\left(\alpha^{2}+X^{2}-Z_{0}^{2}\right)+j 2 Z_{0} X\right\}}{2}+\psi .
$$

\section{Multiband Transmission Lines}

The circuit parameters of a conventional RH TL are series inductance and shunt capacitance. This structure has a linear phase response, and only a RH phase shift or phase delay exists. However, the dual structure of a RH TL or LH TL, of which the circuit parameters are series capacitance and shunt inductance, has a nonlinear phase response, and only a LH phase response or phase advance is supported. Because of a parasitic RH TL, a pure LH TL does not exist in the real world, and a combination of a RH TL and a LH TL, a CRLH TL, has been actively investigated [17]. Fig. 3 shows the circuit model and phase response of a CRLH TL. A CRLH TL shows interesting characteristics in the balanced condition, where the series resonant and shunt resonant frequencies are equal. In the balanced condition of a CRLH TL, the phase response and characteristic impedance are simply represented as, 


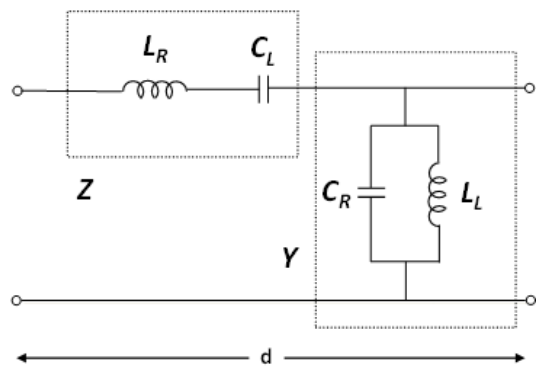

(a)

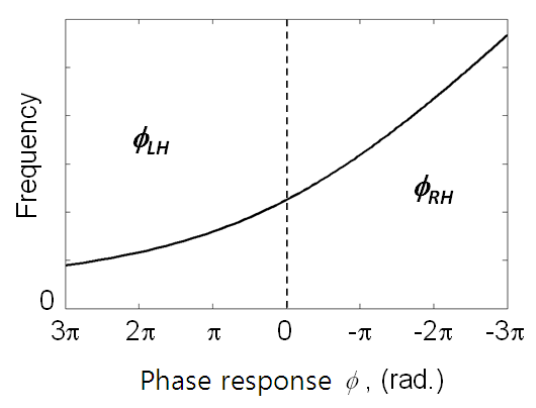

(b)

Fig. 3. (a) Circuit diagram of a composite right/left-handed transmission line (CRLH TL) unit cell and (b) phase response of a CRLH TL in the balanced case.

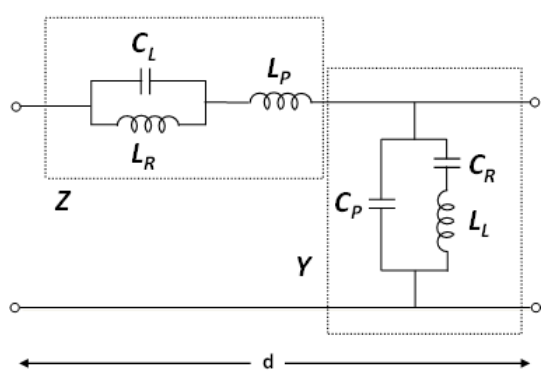

(a)

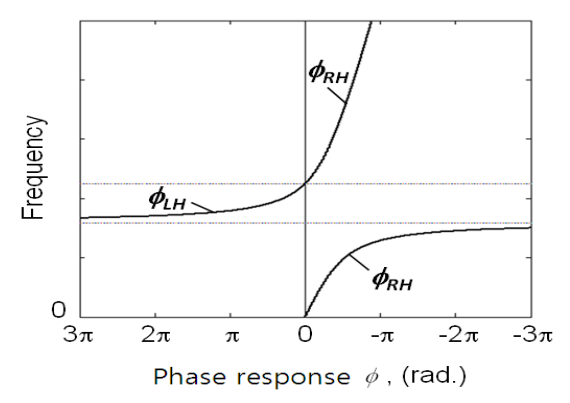

(b)

Fig. 4. (a) Circuit model of a double-Lorentz transmission line (DL TL) unit cell and (b) phase response of a DL TL in the balanced case. Source [13], reproduced by permission of (C) 2012 EuMA.

$$
\phi(\omega)=-N_{C}\left[\omega \sqrt{L_{R} C_{R}}-\frac{1}{\omega \sqrt{L_{L} C_{L}}}\right]
$$

$$
Z_{C}=\sqrt{\frac{L_{R}}{C_{R}}}=\sqrt{\frac{L_{L}}{C_{L}}}
$$

where $N_{C}$ is the number of CRLH TL unit cells and $\omega$ is the angular frequency. A CRLH TL has a dual-band characteristic: two desired phase responses, $\phi_{1}$ and $\phi_{2}$, can be obtained at two frequencies, $\omega_{1}$ and $\omega_{2}$, using a CRLH TL. Phase shift $\phi_{1}$ at frequency $\omega_{1}$ and phase shift $\phi_{2}$ at frequency $\omega_{2}$ bring two equations using (3). Because of the matching-to-termination impedance $Z_{0}$, (4) makes two other equations. So, there are four equations with four unknowns: $L_{R}, C_{R}, L_{L}$, and $C_{L}$. These four circuit parameters are presented with closed forms by modifying four equations which come from (3) and (4) [17].

A dual-CRLH (D-CRLH) TL has not only the dual characteristics of a CRLH TL but also has the dual structure of a CRLH TL, which has the parallel LC in series branch and series LC in shunt branch [18]. A DL TL is a combination of a D-CRLH TL and a parasitic RH TL, of which circuit parameters are series inductance $\left(L_{p}\right)$ and shunt capacitance $\left(C_{p}\right)$. Fig. 4(a) shows a circuit diagram of a DL TL. The phase response of a DL TL, shown in Fig. 4(b), is similar to that of a DCRLH TL, but the existence of a RH phase shift region at a higher frequency band is a significant difference. A DL TL appears as a RH TL at low frequencies because components $L_{R}, L_{P}, C_{R}$, and $C_{p}$ are dominant. On the contrary, if both impedance $\mathrm{Z}$ and admittance $\mathrm{Y}$ could have negative values at a certain frequency band, the DL TL would show CRLH-like behavior, because the effect of $L_{R}$ and $C_{R}$ in the structure is reduced at higher frequency. However, if both $\mathrm{Z}$ and $\mathrm{Y}$ do not support negative values in the frequency band, then the DL TL does not have a LH characteristic.

The phase response and characteristic impedance of a balanced DL TL are presented as (5) and (6), respectively [19].

$$
\begin{aligned}
& \phi(\omega)=-N_{D}\left[\omega \sqrt{L_{P} C_{P}}+\sqrt{\frac{C_{P}}{L_{P}}} \frac{\omega L_{R}}{1-\omega^{2} L_{R} C_{L}}\right] \\
& Z_{\mathrm{C}}=\sqrt{\frac{L_{P}}{C_{P}}}=\sqrt{\frac{L_{L}}{C_{L}}}=\sqrt{\frac{L_{R}}{C_{R}}} .
\end{aligned}
$$

where $N_{D}$ is the number of DL TL unit cells. Because a DL TL has a tri-band characteristic, three desired phase responses, $\phi_{1}, \phi_{2}$, and $\phi_{3}$, can be obtained at three frequencies, $\omega_{1}, \omega_{2}$, and $\omega_{3}$. This results in three equations using (5). Because of the matching-to-termination impedance $Z_{0}$, (6) makes three other equations. Hence there are six equations with six unknowns, $L_{P}, C_{P}, L_{R}$, $C_{R}, L_{L}$, and $C_{L}$. Solving these simultaneous equations 
gives circuit parameters of a DL TL satisfying tri-band characteristic.

\section{Multiplexers}

In order to verify the design concepts, star junction type multiplexers and manifold junction type multiplexers are designed and fabricated. A RT/Duroid 5870 substrate $\left(\varepsilon_{r}=2.33\right.$, height $\left.=0.787 \mathrm{~mm}\right)$ and commercial surface acoustic wave filters are used for multiplexers. Four filters (having 881.5, 942.5, 1,575, and 1,842.5 $\mathrm{MHz}$ center frequencies) are made by Panasonic while the manufacturers of other filters, with 2,140 and 2,450 $\mathrm{MHz}$ center frequencies, are EPCOS and Murata, respectively.

\section{Star Junction Type Multiplexers}

The triplexer based on dual-band isolation circuits is shown in Fig. 5(a). Because of using commercial filters, this triplexer has smaller size than the previous work [12]. The center frequencies of commercial filters are $881.5,1,575$, and $1,842.5 \mathrm{MHz}$, and each isolation circuit allows signal path at the center frequency of its filter. However, it rejects signal at two operating frequencies of other isolation circuits. For designing CRLH TLs used on dual-band isolation circuits, two target input impedances of each filter are necessary and they are represented in Table 1. After calculating required two phases from two input impedances with (2c) or (2d), the circuit parameters of each CRLH TL can be solved from (3) and (4). The results are summarized in Table 1. For a CRLH TL, lumped elements and microstrip lines are used. A LH part of a CRLH TL was realized using lumped elements whereas a microstrip line was used for a RH TL part. Fig. 6(a)-(c) show the simulated and measured results of the triplexer. Fig. 6(a) shows the insertion losses, which are less than 2.2 dB. Fig. 6(b) shows the return losses better than $15.1 \mathrm{~dB}$, and the isolation is greater than $33.5 \mathrm{~dB}$ in Fig. 6(c).

Fig. 5(b) shows the fabricated quadruplexer based on tri-band isolation circuits [13]. The center frequencies are $942.5,1,575,2,140$, and $2,450 \mathrm{MHz}$. In a manner similar to that of a dual-band isolation circuit, a DL TL for a tri-band isolation circuit can be designed by getting required phases from input impedances of a filter used on the isolation circuit. Then, circuit parameters of the DL TL are solved from (5) and (6) with the required phase responses and corresponding frequencies. Table 2 shows the input impedances of each filter, the required phase responses, and the circuit parameters of the DL TL satisfying tri-band isolation characteristic. A D-CRLH part of a DL TL was realized using lumped ele-

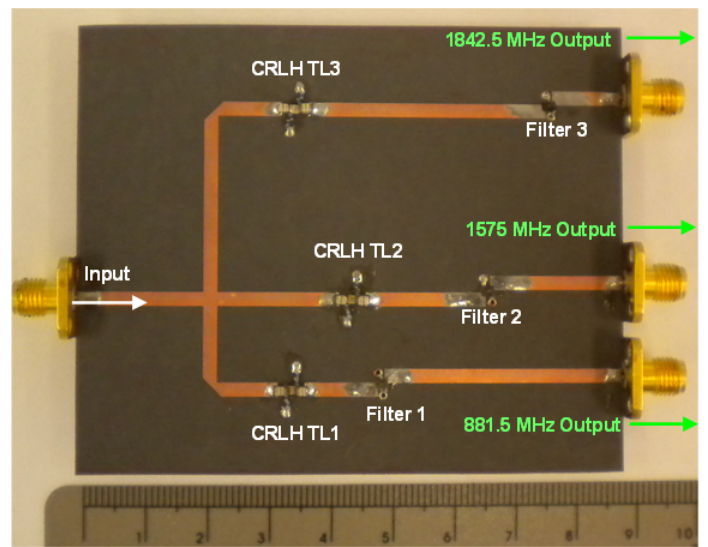

(a)

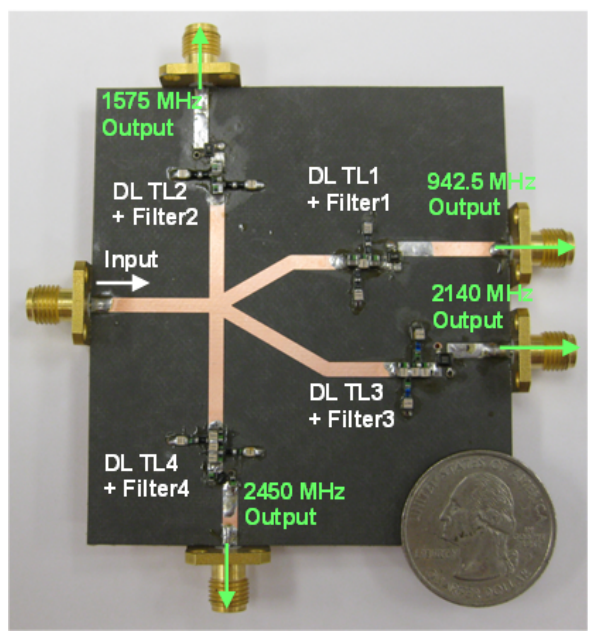

(b)

Fig. 5. Fabricated star-junction multiplexers based on isolation circuits: (a) triplexer and (b) quadruplexer. (b) is from [13], reproduced by permission of (c) 2012 EuMA.

ments while a microstrip line was used for a $\mathrm{RH}$ TL part. The simulated and measured results of the quadruplexer are shown in Fig. 6(d)-(f). The worst case of insertion losses shown in Fig. 6(d) is 4.07 dB. This result can be improved by further adjusting the reactance of lumped elements. Insertion losses of other output ports are less than $2.9 \mathrm{~dB}$. Fig. 6(e) shows the return losses larger than $11.3 \mathrm{~dB}$, and the isolation is larger than $27.5 \mathrm{~dB}$ in Fig. 6(f).

\section{Manifold Junction Type Multiplexers}

Fig. 7(a) shows the picture of the manifold junction triplexer based on dual-band isolation circuits [14]. Its center frequencies are 881.5, 1,575, and 1,842.5 MHz. The design process of a dual-band isolation circuit based on a CRLH TL is same with that of the star junction triplexer shown in previous section. However, the special isolation circuit based on RH TLs has different ope- 


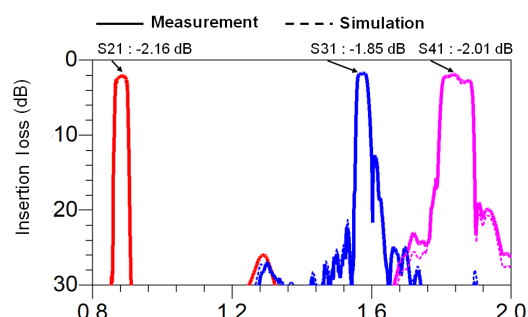

(a)

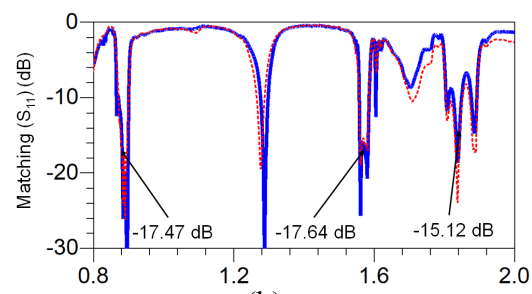

(b)

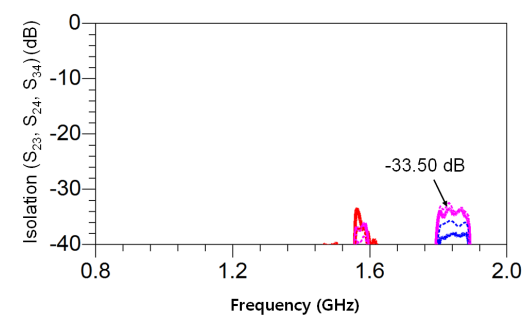

(c)

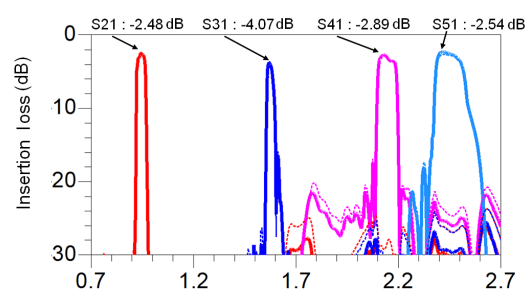

(d)

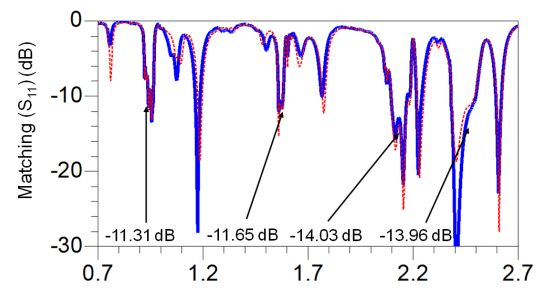

(e)

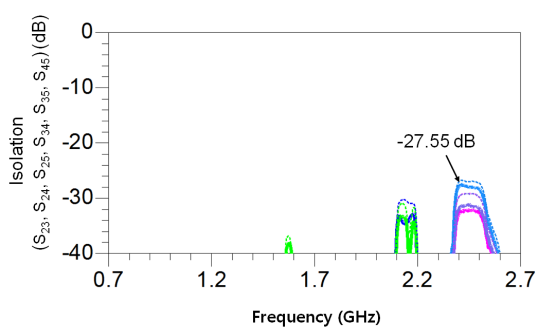

(f)

Fig. 6. The star-junction triplexer results: (a) insertion loss, (b) matching at port 1, and (c) isolation. The starjunction quadruplexer results: (d) insertion loss, (e) matching at port 1 , and (f) isolation. (d), (e), and (f) are from [13], reproduced by permission of (C) 2012 EuMA. ration concept and design method. At 1,575 $\mathrm{MHz}$, the combined phase response of RH TL1 and RH TL2 should satisfy the condition that the input impedance of the special isolation circuit (consisting of two RH TLs and $881.5 \mathrm{MHz}$ filter) is infinite. Similarly, the phase response of RH TL2 should be determined having open condition with the filter at 1,842.5 MHz. Table 3 shows the summary of designed isolation circuits. Fig. 8(a)(c) show the simulated and measured results of the triplexer. The insertion losses shown in Fig. 8(a) are less than $2.1 \mathrm{~dB}$. Fig. 8(b) shows the return losses better than $14.3 \mathrm{~dB}$, and the isolation is greater than $32.4 \mathrm{~dB}$ in Fig. 8(c).

The manifold junction quadruplexer based on tri-band isolation circuits is shown in Fig. 7(b) [15]. The design process is similar with the triplexer case, except that the quadruplexer uses DL TLs and three RH TLs instead of two RH TLs. Table 4 shows the design summary of the quadruplexer. Fig. 8(d)-(f) show the simulated and measured results of the quadruplexer, and simulation results are well matched with measured results. Fig. 8(d) shows the insertion losses, which are less than $4.1 \mathrm{~dB}$.

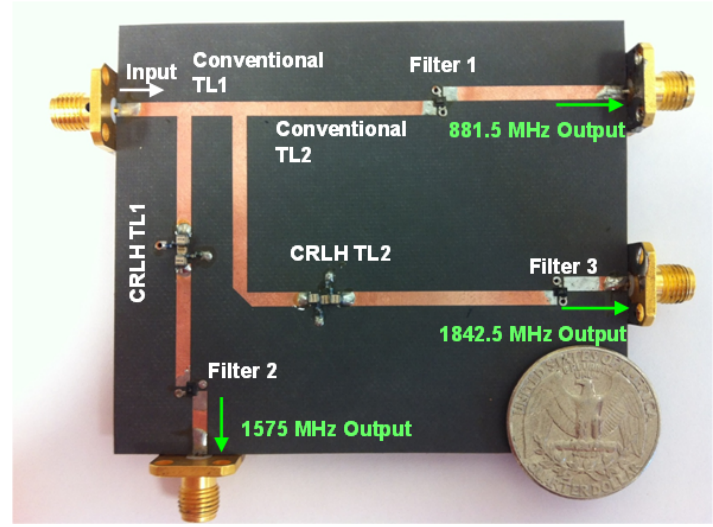

(a)

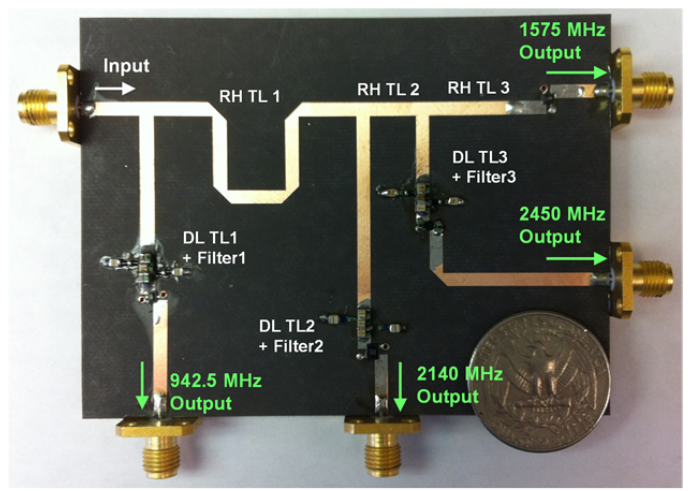

(b)

Fig. 7. Fabricated manifold-junction multiplexers based on isolation circuits: (a) triplexer and (b) quadruplexer. (a) is from [14], reproduced by permission of (c) 2012 IEEE. (b) is from [15]. 


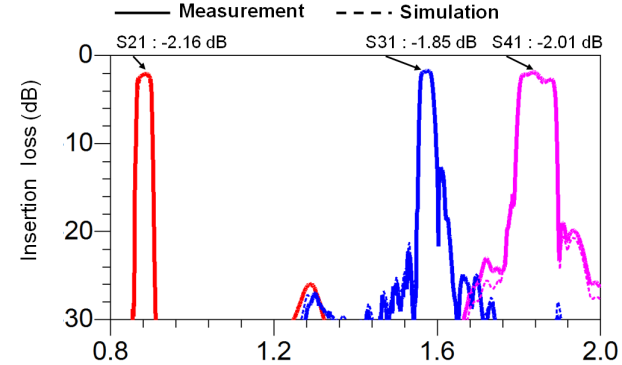

(a)

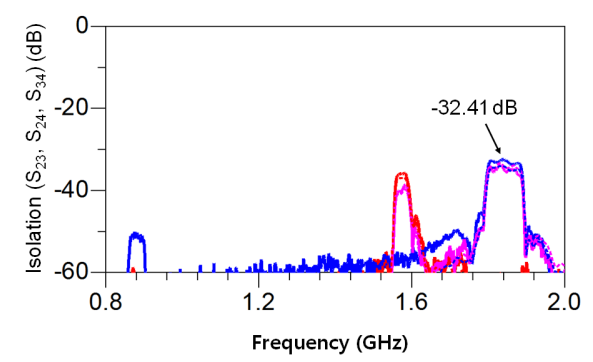

(c)

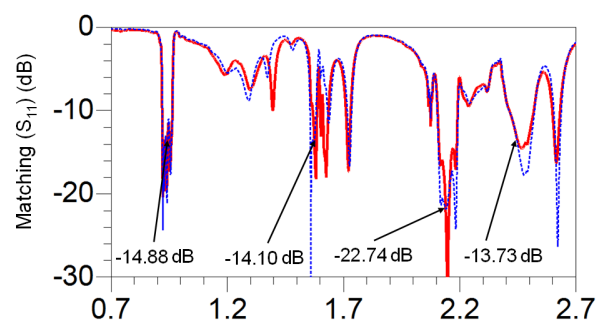

(e)

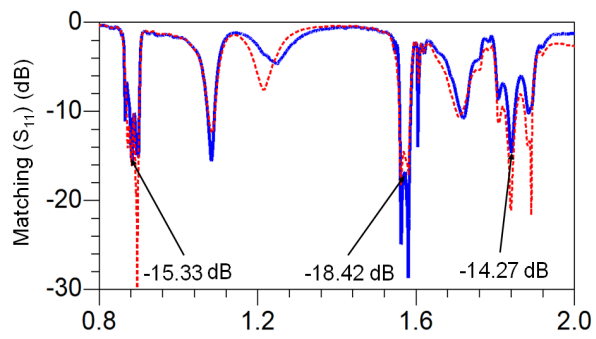

(b)

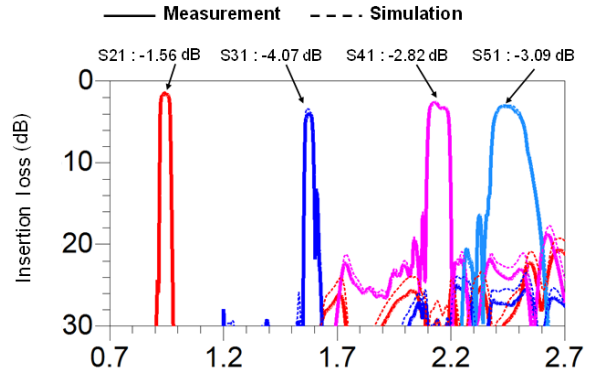

(d)

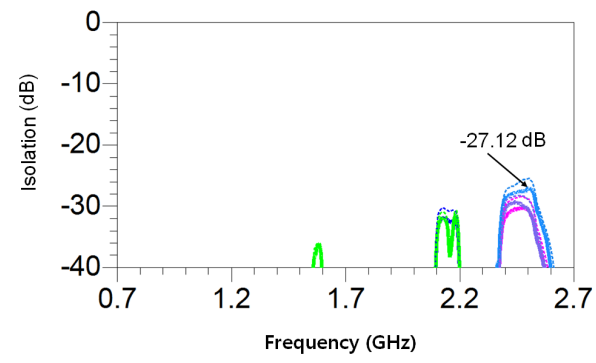

(f)

Fig. 8. The manifold junction triplexer results: (a) insertion loss, (b) matching at port 1, and (c) isolation. The manifold junction quadruplexer results: (d) insertion loss, (e) matching at port 1, and (f) isolation. (a), (b), and (c) are from [14], reproduced by permission of (c) 2012 IEEE. (d), (e), and (f) are from [15].

Table 1. Design summary of the star junction triplexer

\begin{tabular}{|c|c|c|c|c|c|c|c|c|}
\hline & \multirow{2}{*}{\multicolumn{2}{|c|}{ Impedance of filter $(\Omega)$}} & \multirow{2}{*}{\multicolumn{2}{|c|}{$\begin{array}{l}\text { Required phase } \\
\text { delay/advance (deg.) }\end{array}$}} & \multicolumn{4}{|c|}{$\begin{array}{c}\text { Circuit parameter of CRLH TL } \\
(L, \mathrm{nH} ; C, \mathrm{pF})\end{array}$} \\
\hline & & & & & $L_{R}$ & $C_{R}$ & $L_{L}$ & $C_{L}$ \\
\hline $\begin{array}{l}881.5 \mathrm{MHz} \\
\text { Isolation circuit }\end{array}$ & $\begin{array}{c}1,575 \mathrm{MHz} \\
-j(25.25)\end{array}$ & $\begin{array}{c}1,842.5 \mathrm{MHz} \\
-j(18.20)\end{array}$ & $\begin{array}{c}\phi_{11} \\
-101.80\end{array}$ & $\begin{array}{c}\phi_{12} \\
-126.30\end{array}$ & 5.5 & 2.2 & 25.3 & 10.1 \\
\hline $\begin{array}{l}1,575 \mathrm{MHz} \\
\text { Isolation circuit }\end{array}$ & $\begin{array}{c}881.5 \mathrm{MHz} \\
-j(58.65)\end{array}$ & $\begin{array}{l}1,842.5 \mathrm{MHz} \\
-j(25.55)\end{array}$ & $\begin{array}{c}\phi_{21} \\
40.45\end{array}$ & $\begin{array}{c}\phi_{22} \\
-117.07\end{array}$ & 6.67 & 2.67 & 8.27 & 3.31 \\
\hline $\begin{array}{l}1,842.5 \mathrm{MHz} \\
\text { Isolation circuit }\end{array}$ & $\begin{array}{c}881.5 \mathrm{MHz} \\
-j(58.70)\end{array}$ & $\begin{array}{c}1,575 \mathrm{MHz} \\
-j(23.75)\end{array}$ & $\begin{array}{c}\phi_{31} \\
40.42\end{array}$ & $\begin{array}{c}\phi_{32} \\
-115.41\end{array}$ & 8.86 & 3.54 & 6.77 & 2.71 \\
\hline
\end{tabular}

CRLH $\mathrm{TL}=$ composite right/left-handed transmission line.

Fig. 8(e) shows that the return losses are better than $13.7 \mathrm{~dB}$, and the isolation is greater than $27.1 \mathrm{~dB}$ as shown in Fig. 8(f).

\section{Conclusions}

Multiplexers based on isolation circuits are presented.
The concepts, theories, and design processes have been explained to help the reader design and fabricate proposed multiplexers. These multiplexers have significant advantages. A straightforward and easy design process and the freedom to choose any filters without modification are attractive to a system designer. In this paper, two different types of triplexers and two types of quad- 
Table 2. Design summary of the star junction quadruplexer

\begin{tabular}{|c|c|c|c|c|c|c|c|c|c|c|c|c|}
\hline & \multirow{2}{*}{\multicolumn{3}{|c|}{ Impedance of filter $(\Omega)$}} & \multirow{2}{*}{\multicolumn{3}{|c|}{$\begin{array}{l}\text { Required phase } \\
\text { delay/advance (deg.) }\end{array}$}} & \multicolumn{6}{|c|}{$\begin{array}{c}\text { Circuit parameter of DL TL } \\
(L, \mathrm{nH} ; C, \mathrm{pF})\end{array}$} \\
\hline & & & & & & & $L_{R}$ & $C_{R}$ & $L_{L}$ & $C_{L}$ & $L_{p}$ & $C_{p}$ \\
\hline $\begin{array}{l}942.5 \mathrm{MHz} \\
\text { Isolation circuit }\end{array}$ & $\begin{array}{c}1,575 \mathrm{MHz} \\
-j(27.75)\end{array}$ & $\begin{array}{c}2,140 \mathrm{MHz} \\
-j(13.75)\end{array}$ & $\begin{array}{c}2,450 \mathrm{MHz} \\
-j(8.10)\end{array}$ & $\begin{array}{c}\phi_{11} \\
60.90\end{array}$ & $\begin{array}{c}\phi_{12} \\
-90.41\end{array}$ & $\begin{array}{c}\phi_{13} \\
-114.19\end{array}$ & 1.80 & 0.72 & 16.18 & 6.47 & 7.50 & 3.00 \\
\hline $\begin{array}{l}1,575 \mathrm{MHz} \\
\text { Isolation circuit }\end{array}$ & $\begin{array}{c}942.5 \mathrm{MHz} \\
-j(54.25)\end{array}$ & $\begin{array}{c}2,140 \mathrm{MHz} \\
-j(16.95)\end{array}$ & $\begin{array}{c}2,450 \mathrm{MHz} \\
-j(11.30)\end{array}$ & $\begin{array}{c}\phi_{21} \\
-137.33\end{array}$ & $\begin{array}{c}\phi_{22} \\
-98.82\end{array}$ & $\begin{array}{c}\phi_{23} \\
-117.76\end{array}$ & 2.75 & 1.10 & 20.43 & 8.17 & 7.31 & 2.92 \\
\hline $\begin{array}{l}\text { 2,140 } \mathrm{MHz} \\
\text { Isolation circuit }\end{array}$ & $\begin{array}{c}942.5 \mathrm{MHz} \\
-j(90.60)\end{array}$ & $\begin{array}{c}1,575 \mathrm{MHz} \\
-j(45.15)\end{array}$ & $\begin{array}{c}2,450 \mathrm{MHz} \\
-j(27.15)\end{array}$ & $\begin{array}{c}\phi_{31} \\
-151.12\end{array}$ & $\begin{array}{c}\phi_{32} \\
47.89\end{array}$ & $\begin{array}{c}\phi_{33} \\
-118.66\end{array}$ & 6.23 & 2.49 & 6.01 & 2.4 & 9.17 & 3.67 \\
\hline $\begin{array}{l}2,450 \mathrm{MHz} \\
\text { Isolation circuit }\end{array}$ & $\begin{array}{c}942.5 \mathrm{MHz} \\
j(18.30) \\
\end{array}$ & $\begin{array}{c}1,575 \mathrm{MHz} \\
j(44.70) \\
\end{array}$ & $\begin{array}{c}2,140 \mathrm{MHz} \\
j(332.65) \\
\end{array}$ & $\begin{array}{c}\phi_{41} \\
-69.89 \\
\end{array}$ & $\begin{array}{c}\phi_{42} \\
131.84\end{array}$ & $\begin{aligned} & \phi_{43} \\
&- 2.88 \\
&\end{aligned}$ & 3.92 & 1.57 & 8.24 & 3.29 & 3.12 & 1.25 \\
\hline
\end{tabular}

Source [13], reproduced by permission of (c) 2012 EuMA.

$\mathrm{DL} \mathrm{TL}=$ double-Lorentz transmission line.

Table 3. Design summary of the manifold junction triplexer

\begin{tabular}{|c|c|c|c|c|c|c|c|c|}
\hline & \multirow{2}{*}{\multicolumn{2}{|c|}{ Impedance of filter $(\Omega)$}} & \multirow{2}{*}{\multicolumn{2}{|c|}{$\begin{array}{l}\text { Required phase delay/ } \\
\text { advance (deg.) }\end{array}$}} & \multicolumn{4}{|c|}{$\begin{array}{l}\text { Circuit parameter of CRLH TL } \\
(L, \mathrm{nH} ; C, \mathrm{pF})\end{array}$} \\
\hline & & & & & $L_{R}$ & $C_{R}$ & $L_{L}$ & $C_{L}$ \\
\hline $\begin{array}{c}1,575 \mathrm{MHz} \\
\text { Isolation circuit }\end{array}$ & $\begin{array}{c}881.5 \mathrm{MHz} \\
-j(58.65)\end{array}$ & $\begin{array}{c}1,842.5 \mathrm{MHz} \\
-j(25.55)\end{array}$ & $\begin{array}{c}\phi_{21} \\
40.45\end{array}$ & $\begin{array}{c}\phi_{22} \\
-117.07\end{array}$ & 6.67 & 2.67 & 8.27 & 3.31 \\
\hline $\begin{array}{c}1,842.5 \mathrm{MHz} \\
\text { Isolation circuit }\end{array}$ & $\begin{array}{c}881.5 \mathrm{MHz} \\
-j(58.70)\end{array}$ & $\begin{array}{c}1,575 \mathrm{MHz} \\
-j(23.75)\end{array}$ & $\begin{array}{c}\phi_{31} \\
40.42\end{array}$ & $\begin{array}{c}\phi_{32} \\
-115.41\end{array}$ & 8.86 & 3.54 & 6.77 & 2.71 \\
\hline $\begin{array}{c}881.5 \mathrm{MHz} \\
\text { Isolation circuit }\end{array}$ & $\begin{array}{c}1,575 \mathrm{MHz} \\
-j(25.25)\end{array}$ & $\begin{array}{c}1,842.5 \mathrm{MHz} \\
-j(18.20)\end{array}$ & $\begin{array}{c}\phi_{11} \\
-116.79\end{array}$ & $\begin{array}{c}\phi_{12} \\
-110.00\end{array}$ & - & - & - & - \\
\hline
\end{tabular}

Source [14], reproduced by permission of (C) 2012 IEEE.

CRLH TL=composite right/left-handed transmission line.

Table 4. Design summary of the manifold junction quadruplexer

\begin{tabular}{|c|c|c|c|c|c|c|c|c|c|c|c|c|}
\hline & \multicolumn{3}{|c|}{ Impedance of filter $(\Omega)$} & \multirow{2}{*}{\multicolumn{3}{|c|}{$\begin{array}{c}\text { Required phase delay/advance } \\
\text { (deg.) }\end{array}$}} & \multicolumn{6}{|c|}{$\begin{array}{l}\text { Circuit parameter of DL TL } \\
\qquad(L, \mathrm{nH} ; C, \mathrm{pF})\end{array}$} \\
\hline & & & & & & & $L_{R}$ & $C_{R}$ & $L_{L}$ & $C_{L}$ & $L_{p}$ & $C_{p}$ \\
\hline $\begin{array}{l}942.5 \mathrm{MHz} \\
\text { Isolation circuit }\end{array}$ & $\begin{array}{l}1575 \mathrm{MHz} \\
-j(27.75)\end{array}$ & $\begin{array}{c}2,140 \mathrm{MHz} \\
-j(13.75)\end{array}$ & $\begin{array}{l}2,450 \mathrm{MHz} \\
-j(8.10)\end{array}$ & $\begin{array}{c}\phi_{11} \\
60.90\end{array}$ & $\begin{array}{c}\phi_{12} \\
-90.41\end{array}$ & $\begin{array}{c}\phi_{13} \\
-114.19\end{array}$ & 1.80 & 0.72 & 16.18 & 6.47 & 7.50 & 3.00 \\
\hline $\begin{array}{l}2,140 \mathrm{MHz} \\
\text { Isolation circuit }\end{array}$ & $\begin{array}{c}942.5 \mathrm{MHz} \\
-j(90.60)\end{array}$ & $\begin{array}{c}1,575 \mathrm{MHz} \\
-j(45.15)\end{array}$ & $\begin{array}{c}2,450 \mathrm{MHz} \\
-j(27.15)\end{array}$ & $\begin{array}{c}\phi_{31} \\
-151.12\end{array}$ & $\begin{array}{c}\phi_{32} \\
47.89\end{array}$ & $\begin{array}{c}\phi_{33} \\
-118.66\end{array}$ & 6.23 & 2.49 & 6.01 & 2.4 & 9.17 & 3.67 \\
\hline $\begin{array}{l}2,450 \mathrm{MHz} \\
\text { Isolation circuit }\end{array}$ & $\begin{array}{c}942.5 \mathrm{MHz} \\
j(18.30)\end{array}$ & $\begin{array}{c}1,575 \mathrm{MHz} \\
j(44.70)\end{array}$ & $\begin{array}{c}2,140 \mathrm{MHz} \\
j(332.65)\end{array}$ & $\begin{aligned} & \phi_{41} \\
- & 69.89\end{aligned}$ & $\begin{array}{c}\phi_{42} \\
131.84\end{array}$ & $\begin{array}{c}\phi_{43} \\
-2.88\end{array}$ & 3.92 & 1.57 & 8.24 & 3.29 & 3.12 & 1.25 \\
\hline $\begin{array}{l}1,575 \mathrm{MHz} \\
\text { Isolation circuit }\end{array}$ & $\begin{array}{c}942.5 \mathrm{MHz} \\
-j(54.25)\end{array}$ & $\begin{array}{l}2,140 \mathrm{MHz} \\
-j(16.95)\end{array}$ & $\begin{array}{c}2,450 \mathrm{MHz} \\
-j(11.30)\end{array}$ & $\begin{array}{c}\phi_{21} \\
-137.30\end{array}$ & $\begin{array}{c}\phi_{22} \\
-108.80\end{array}$ & $\begin{array}{c}\phi_{23} \\
-102.80\end{array}$ & - & - & - & - & - & - \\
\hline
\end{tabular}

Source [15].

$\mathrm{DL} \quad \mathrm{TL}=$ double-Lorentz transmission line.

ruplexers are designed and fabricated. The measured results of each multiplexer show good agreement with the simulation results.
This work was supported by the Panasonic Corporation. 


\section{References}

[1] G. Macchiarella, "Synthesis of star-junction multiplexer," IEEE Microwave Magazine, vol. 12, no. 6, pp. 101-109, Oct. 2011.

[2] G. Macchiarella and S. Tamiazzo, "Synthesis of starjunction multiplexers," IEEE Transactions on Microwave Theory and Techniques, vol. 58, no. 12, pp. 3732-3741, Dec. 2010.

[3] R. J. Cameron and M. Yu, "Design of manifold-coupled multiplexers," IEEE Microwave Magazine, vol. 8, no. 5, pp. 46-59, Oct. 2007.

[4] E. G. Cristal and G. L. Matthaei, "A technique for the design of multiplexers having contiguous channels," IEEE Transactions on Microwave Theory and Techniques, vol. 12, no. 1, pp. 88-93, Jan. 1964.

[5] J. D. Rhodes and R. Levy, "A generalized multiplexer theory," IEEE Transactions on Microwave Theory and Techniques, vol. 27, no. 2, pp. 99-111, Feb. 1979.

[6] K. L. Wu and W. Meng, "A direct synthesis approach for microwave filters with a complex load and its application to direct diplexer design," IEEE Transactions on Microwave Theory and Techniques, vol. 55, no. 5, pp. 1010-1017, May 2007.

[7] J. D. Rhodes and R. Levy, "Design of general manifold multiplexers," IEEE Transactions on Microwave Theory and Techniques, vol. 27, no. 2, pp. 111-123, Feb. 1979.

[8] A. E. Atia, "Computer aided design of waveguide multiplexers," IEEE Transactions on Microwave Theory and Techniques, vol. 22, no. 3, pp. 322-336, Mar. 1974.

[9] M. A. Ismail, D. Smith, A. Panariello, Y. Wang, and M. Yu, "EM-based design of large-scale dielectric resonator filters and multiplexers by space mapping," IEEE Transactions on Microwave Theory and Techniques, vol. 52, no. 1, pp. 386-392, Jan. 2004.

[10] I. H. Lin, M. DeVincentis, C. Caloz, and T. Itoh, "Arbitrary dual-band components using composite right/left-handed transmission lines," IEEE Transactions on Microwave Theory and Techniques, vol.
52, no. 4, pp. 1142-1149, Apr. 2004.

[11] A. Rennings, T. Liebig, C. Caloz, and I. Wolff, "Double-Lorentz transmission line metamaterial and its application to tri-band devices," in Proceedings of the IEEE MTT-S International Microwave Symposium, Honolulu, HI, 2007, pp. 1427-1430.

[12] H. Lee and T. Itoh, "Dual band isolation circuits based on CRLH transmission lines for triplexer application," in Proceedings of the Asia-Pacific Microwave Conference, Melbourne, Australia, 2011, pp. 542-545.

[13] H. Lee and T. Itoh, "Tri-band isolation circuits based on double-Lorentz transmission lines for quadruplexers," in Proceedings of the 42nd European Microwave Conference, Amsterdam, the Netherlands, 2012, pp. 585-588.

[14] H. Lee and T. Itoh, "Hybrid combination of dual band isolation circuits based on conventional and CRLH transmission lines for triplexers," in Proceedings of the IEEE MTT-S International Microwave Symposium, Montreal, Canada, 2012.

[15] H. Lee and T. Itoh, "Hybrid combination of triband isolation circuits based on conventional and double-Lorentz transmission lines for quadruplexers," in Proceedings of the 2013 URSI International Symposium on Electromagnetic Theory, Hiroshima, Japan, 2013, pp. 393-396.

[16] D. M. Pozar, Microwave Engineering, 3rd ed. Hoboken, NJ: John Willey \& Sons, 2005.

[17] C. Caloz and T. Itoh, Electromagnetic Metamaterials: Transmission Line Theory and Microwave Applications. Hoboken, NJ: John Willey \& Sons, 2006.

[18] C. Caloz, "Dual composite right/left-handed (D-CRLH) transmission line metamaterial," IEEE Microwave and Wireless Components Letters, vol. 16, no. 11, pp. 585-587, Nov. 2006.

[19] H. Lee and S. Nam, "Triband branch line coupler using double-Lorentz transmission lines," Microwave and Optical Technology Letters, vol. 50, no. 5, pp. 1174-1177, May 2008. 


\section{Hanseung Lee}

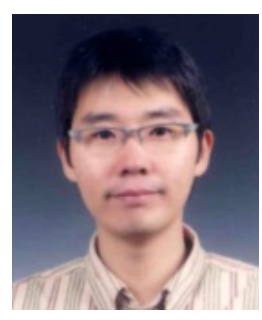

received the B.S. degree from the Korea University, Seoul, Republic of Korea, in 2006, and the M.S. degree from the Seoul National University, in 2008, both in electrical engineering and he is currently working toward the Ph.D. degree in electrical engineering from the University of California at Los Angeles (UCLA). Since September 2010, he has been a Graduate Student Researcher at the Microwave Electronics Laboratory, UCLA. His research interests include design of a passive circuit and an antenna for $\mathrm{RF} /$ microwave, and applications of metamaterials. Mr. Lee is the recipient of Asia-Pacific Microwave Conference (APMC) 2011 Prize.

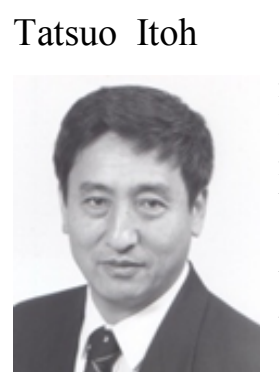

received the Ph.D. degree in Electrical Engineering from the University of Illinois, Urbana in 1969. After working for University of Illinois, SRI and University of Kentucky, he joined the faculty at The University of Texas at Austin in 1978, where he became a Professor of Electrical Engineering in 1981. In September 1983, he was selected to hold the Hayden Head Centennial Professorship of Engineering at The University of Texas. In January 1991, he joined the University of California, Los Angeles as Professor of Electrical Engineering and holder of the TRW Endowed Chair in Microwave and Millimeter Wave Electronics (currently Northrop Grumman Endowed Chair). He received a number of awards including IEEE Third Millennium Medal in 2000, and IEEE MTT Distinguished Educator Award in 2000. He was elected to a member of National Academy of Engineering in 2003. In 2011, he received Microwave Career Award from IEEE MTT Society. Dr. Itoh is a Fellow of the IEEE, a member of the Institute of Electronics and Communication Engineers of Japan, and Commissions $\mathrm{B}$ and D of SNC/URSI. He served as the Editor of IEEE Transactions on Microwave Theory and Techniques for 1983 - 1985. He was President of the Microwave Theory and Techniques Society in 1990. He was the Editor-in-Chief of IEEE Microwave and Guided Wave Letters from 1991 through 1994. He was elected as an Honorary Life Member of MTT Society in 1994. He was the Chairman of Commission D of International URSI for 1993-1996, the Chairman of Commission D of International URSI for 1993-1996. He serves on advisory boards and committees of a number of organizations. He served as Distinguished Microwave Lecturer on Microwave Applications of Metamaterial Structures of IEEE MTT-S for 2004-2006. He has 420 journal publications, 820 refereed conference presentations and has written 48 books/ book chapters in the area of microwaves, millimeter-waves, antennas and numerical electromagnetics. He generated 75 Ph.D. students. 